\title{
A multiplicity result for the non-homogeneous Klein-Gordon-Maxwell system in rotationally symmetric bounded domains
}

Yuhu Wu ${ }^{1 *}$ and Bin Ge

\section{"Correspondence:}

wuyuhu51@gmail.com

1 Department of Mathematics,

Harbin University of Science and

Technology, Harbin, 150080, PR

China

Full list of author information is

available at the end of the article

\begin{abstract}
This paper is concerned with the Klein-Gordon equation coupled with the Maxwell equation in the rotationally symmetric bounded domains when a non-homogeneous term breaks the symmetry of the associated functional. Under some suitable assumption on nonlinear perturbation, we obtain infinitely many radially symmetric solutions to the non-homogeneous Klein-Gordon-Maxwell system.
\end{abstract}

\section{Introduction}

This paper is concerned with the existence and multiplicity of solutions for a class of KleinGordon-Maxwell (KGM for short) systems in rotationally symmetric bounded domains. We fix $0 \leq \rho<\infty$ and define

$$
\Omega=\Omega_{\rho}:=\operatorname{int}\left\{x \in R^{N}(N=3,4 \text { or } 5): \rho \leq|x|<\rho+1\right\},
$$

when $\rho=0, \Omega=\Omega_{0}$ is a ball and when $\rho>0, \Omega=\Omega_{\rho}$ is an annulus in $R^{N}(N=3,4,5)$.

We consider the KGM system in this type symmetric bounded domains (the model in a bounded domain was introduced by D'Avenia et al. [1])

$$
\left\{\begin{array}{l}
-\Delta u-(q \phi-\omega)^{2} u+m^{2} u=f(|x|, u)+g(x), \\
\Delta \phi=4 \pi q(q \phi-\omega) u^{2}
\end{array} \text { in } \Omega\right.
$$

with Dirichlet boundary conditions

$$
\left\{\begin{array}{l}
u=0, \\
\phi=\zeta
\end{array} \quad \text { on } \partial \Omega\right.
$$

where $m, q$ and $\omega$ are real constants and $\zeta \in C(\partial \Omega)$.

This system appears as a model which describes the nonlinear Klein-Gordon field in a three-dimensional space interacting with the electromagnetic field. Specifically, in 2002 Benci and Fortunato [2] proposed this couplement, and in their subsequent article [3]

\section{Springer}

๑2013 Wu and Ge; licensee Springer. This is an Open Access article distributed under the terms of the Creative Commons Attribution License (http://creativecommons.org/licenses/by/2.0), which permits unrestricted use, distribution, and reproduction in any medium, provided the original work is properly cited. 
they proved the existence of infinitely many radially symmetric solutions for the KleinGordon-Maxwell system when the nonlinearity exhibits subcritical behavior. D'Aprile and Mugnai [1] established the existence of infinitely many radially symmetric solutions for the subcritical KGM system in $R^{3}$. They extended the interval of definitions of power in the nonlinearity exhibited in [3]. Non-existence results of the KGM system in the whole $R^{3}$ can be found in [4] and [5]. Positive and ground state solutions for the critical KGM system with potentials in $R^{3}$ were obtained in [6]. For related works, see [7-9], and [10].

The KGM system in the case of bounded domains was firstly considered by D'Avenia et al. [11, 12]. They established the existence and multiplicity of solutions to the KGM system in bounded domains under suitable Dirichlet or mixed boundary conditions.

All the above mentioned results are mainly focused on the homogeneous case $g \equiv 0$. However, on the non-homogeneous case $g \neq 0$, only a few results are known for KGM systems. In [13], Chen and Tang proved two different solutions for the non-homogeneous KGM equations in the whole $R^{3}$. Candela and Salvatore [14,15] dealt with multiplicity of solutions to non-homogeneous Schrödinger-Maxwell and Klein-Gordon-Maxwell systems with homogenous boundary conditions in a bounded ball, respectively. Inspired by Candela and Salvatore's results, in this paper we deal with the non-homogeneous KGM system in the special bounded symmetric domains - balls or annuli - and obtain infinitely many radially symmetric solutions by using the variational method.

In the uncoupled case $q=0$, problem (1)-(2) can be split into

$$
\begin{cases}-\Delta u+\left(m^{2}-\omega^{2}\right) u=f(|x|, u)+g(x) & \text { in } \Omega, \\ u=0 & \text { on } \partial \Omega\end{cases}
$$

and

$$
\begin{cases}\Delta \phi=0 & \text { in } \Omega \\ \phi=\zeta & \text { on } \partial \Omega\end{cases}
$$

The existence and uniqueness of solutions of problem (3) and (4) are independent of each other. And it is clear that problem (4) has a unique solution. In this paper, we mainly consider the coupled case $q \neq 0$. In this case, the change of variables

$$
\left\{\begin{array}{l}
u_{q}=\sqrt{4 \pi} q u \\
\varphi_{q}=q \varphi-\omega
\end{array}\right.
$$

transforms system (1)-(2) into

$$
\begin{cases}-\Delta u_{q}+\phi_{q}^{2} u+m^{2} u_{q}=f_{q}\left(|x|, u_{q}\right)+g(x) & \text { in } \Omega, \\ \Delta \phi_{q}=\phi_{q} u_{q}^{2} & \text { in } \Omega, \\ u_{q}=0 & \text { on } \partial \Omega, \\ \phi_{q}=q \zeta-\omega & \text { on } \partial \Omega\end{cases}
$$

with

$$
f_{q}\left(|x|, u_{q}\right)=\sqrt{4 \pi} q f\left(|x|, \frac{u}{\sqrt{4 \pi} q}\right) .
$$


Based on the observation above, we will focus on studying the existence and multiplicity of solutions for problem (5), instead of problem (1)-(2). For the sake of simplicity, from now on, we shall omit the subscript $q$ in problem (5). We assume that the nonlinearity term $f$ in (5) satisfies the following conditions.

$\left(f_{1}\right)$ There exist $a_{1}, a_{2} \geq 0$ and $p \in\left(2, \frac{2 N}{N-2}\right)$ such that $|f(r, u)| \leq a_{1}+a_{2}|u|^{p-1}$.

$\left(\mathrm{f}_{2}\right) f$ is odd with respect to the second variable, that is, for all $r \geq 0, u \in R, f(r,-u)=$ $-f(r, u)$.

$\left(f_{3}\right)$ There exists a constant $s \in(2, p]$ such that for all $r \geq 0, u>0,0<s F(r, u) \leq u f(r, u)$, with $F(r, u)=\int_{0}^{t} f(r, \tau) d \tau$.

And we also assume that the non-homogeneous term $g$ satisfies

( $\left.g_{1}\right) g \in L^{2}(\Omega)$ and $g(|x|)=g(x), \forall x \in \Omega$.

Condition $\left(f_{3}\right)$ is known in the literature as the Ambrosetti-Rabinowitz type condition. The main result we provide in this paper is the following theorem.

Theorem 1.1 Assume that conditions $\left(\mathrm{f}_{1}\right)-\left(\mathrm{f}_{3}\right)$ and $\left(\mathrm{g}_{1}\right)$ hold and

$$
-\frac{s-2}{s}|m|<q \zeta-\omega \leq 0 \quad \text { a.e. } \partial \Omega \text {. }
$$

If $\zeta$ is a radial function on $\partial \Omega$, then system (5) has infinitely many radial solutions $\left(u_{i}, \phi_{i}\right) \in$ $H_{0, r}^{1}(\Omega) \times H_{r}^{1}(\Omega)$ with $\left\|\nabla u_{i}\right\|_{2} \rightarrow+\infty$ and $\left\{\phi_{i}\right\}$ bounded in $L^{\infty}(\Omega)$.

Remark 1.1 Theorem 1.1 can be viewed as a reasonable extension of Theorem 1.1 of [14], where the multiplicity of solutions to the non-homogeneous KGM system with homogenous boundary conditions in a bounded ball of $R^{3}$ can be found.

This paper is organized as follows. In Section 2 we introduce the variational tools: a variational principle, stated as in [3], which allows us to reduce the previous systems to a semilinear elliptic equation in the only variable $u$, and a perturbation method, introduced by Bolle [16, 17], useful for stating our multiplicity results. Finally, in Section 3 we prove our main theorem.

\section{Preliminary results}

In this section, we present the variational framework to deal with problem (5) and also give some preliminaries which are useful later.

To get homogeneous boundary conditions, we change variables as follows:

$$
\varphi=\phi-\phi_{0},
$$

where $\phi_{0}$ is the unique solution of

$$
\begin{cases}\Delta \phi_{0}=0 & \text { in } \Omega, \\ \phi_{0}=q \zeta-\omega & \text { on } \partial \Omega\end{cases}
$$


Then problem (5) can be rewritten as

$$
\begin{cases}-\Delta u-\left(\varphi+\phi_{0}\right)^{2} u+m^{2} u=f(|x|, u)+g(x) & \text { in } \Omega, \\ \Delta \varphi=\left(\varphi+\phi_{0}\right) u^{2} & \text { in } \Omega, \\ u=0, \quad \varphi=0 & \text { on } \partial \Omega .\end{cases}
$$

Remark 2.1 By the maximum principle, (6) implies

$$
-\frac{s-2}{s}|m|<\phi_{0} \leq 0 \quad \text { a.e. } \partial \Omega \text {. }
$$

System (8) contains the Euler-Lagrange equations related to the functional

$$
I: H_{0}^{1}(\Omega) \times H_{0}^{1}(\Omega) \rightarrow R
$$

defined as

$$
\begin{aligned}
I(u, \varphi)= & \frac{1}{2} \int_{\Omega}\left(|\nabla u|^{2}-|\nabla \varphi|^{2}+\left[m^{2}-\left(\varphi+\phi_{0}\right)^{2}\right] u^{2}\right) d x \\
& -\int_{\Omega} F(|x|, u) d x-\int_{\Omega} g u d x .
\end{aligned}
$$

By the standard argument, $I(u, \varphi)$ is $C^{1}$ on $H_{0}^{1}(\Omega) \times H_{0}^{1}(\Omega)$.

The functional $I$ is strongly indefinite, that is, unbounded from below and from above on an infinite dimensional subspace. So we apply a well-known reduction argument (see, e.g., [1]) to avoid this indefiniteness.

Similar to Lemma 2.1 of [4], which deals with the case of the entire domain $R^{3}$, one easily obtains the following auxiliary result. To avoid repetition, the details of the proof, which is mainly based on the Lax-Milgram lemma, are omitted.

Lemma 2.1 For any $u \in H_{0}^{1}(\Omega)$ and for any $h \in H^{-1}(\Omega)$, there exists a unique solution $\varphi:=\left(\Delta-u^{2}\right)^{-1}[h] \in H_{0}^{1}(\Omega)$ of the equation

$$
\begin{cases}\Delta \varphi=u^{2} \varphi+h & \text { in } \Omega, \\ \varphi=0 & \text { on } \partial \Omega .\end{cases}
$$

Moreover, for every $u \in H_{0}^{1}(\Omega)$ and for every $h, k \in H^{-1}(\Omega)$,

$$
\left\langle h,\left(\Delta-u^{2}\right)^{-1}[k]\right\rangle=\left\langle k,\left(\Delta-u^{2}\right)^{-1}[h]\right\rangle,
$$

where $\langle\cdot, \cdot\rangle$ denotes the duality pairing between $H_{1}^{0}(\Omega)$ and $H^{-1}(\Omega)$.

By taking $h=\phi_{0} u^{2}$, we can deduce the following result.

Proposition 2.1 For any $u \in H_{0}^{1}(\Omega)$, there exists unique $\varphi=\varphi_{u} \in H_{0}^{1}(\Omega)$ which satisfies

$$
\begin{cases}\Delta \varphi=\left(\varphi+\phi_{0}\right) u^{2} & \text { in } \Omega, \\ \varphi=0 & \text { on } \partial \Omega .\end{cases}
$$


Furthermore, the map $\Phi: u \in H_{0}^{1}(\Omega) \rightarrow \varphi_{u} \in H_{0}^{1}(\Omega)$ is of class $C^{1}$ and for every $u, v \in$ $H_{0}^{1}(\Omega)$

$$
\left(\Phi^{\prime}[u]\right)[v]=2\left(\Delta-u^{2}\right)^{-1}\left[\left(\varphi_{u}+\phi_{0}\right) u v\right] .
$$

From the above proposition, one directly gets the following assertion.

Corollary 2.1 Let $u \in H_{0}^{1}(\Omega)$ and set $\Psi_{u}:=\left(\Phi^{\prime}[u]\right)[u] \in H_{0}^{1}(\Omega)$. Then $\Psi_{u}$ is a solution to the integral equation

$$
\int_{\Omega} \phi_{0} \Psi_{u} u^{2} d x=\int_{\Omega}\left(\phi_{0}+\varphi_{u}\right) \varphi_{u} u^{2} d x
$$

and

$$
\Psi_{u} \leq 0 .
$$

Since the map $\Phi: u \in H_{0}^{1}(\Omega) \rightarrow \varphi_{u} \in H_{0}^{1}(\Omega)$ is continuously differentiable, we define the reduced $C^{1}$ functional $K(u):=I\left(u, \varphi_{u}\right)$. It is easy to see that the pair $(u, \varphi) \in H_{0}^{1}(\Omega) \times H_{0}^{1}(\Omega)$ is a solution of a critical point for $I$ if and only if $u \in H_{0}^{1}(\Omega)$ is a critical point for $K$ and $\varphi=\Phi[u]$ (see [2]). Hence, we look for critical points of $K$.

Since, by Proposition 2.1, the functional $\varphi_{u}$ satisfies

$$
\left\|\nabla \varphi_{u}\right\|_{2}^{2}=-\int_{\Omega} \varphi_{u}\left(\varphi_{u}+\phi_{0}\right) u^{2} d x
$$

we obtain, for any $u \in H_{0}^{1}(\Omega)$,

$$
\begin{aligned}
K(u)=I\left(u, \varphi_{u}\right)= & \frac{1}{2} \int_{\Omega}\left(|\nabla u|^{2}+\left[m^{2}-\phi_{0}\left(\varphi_{u}+\phi_{0}\right)\right] u^{2}\right) d x \\
& -\int_{\Omega} F(|x|, u) d x-\int_{\Omega} g u d x .
\end{aligned}
$$

According to Lemma 2.1 and Proposition 2.1, for every $u, v \in H_{0}^{1}(\Omega)$, we have

$$
\begin{aligned}
K^{\prime}(v)[u]= & \int_{\Omega} \nabla v \nabla u d x+\left(m^{2}-\phi_{0}^{2}\right) \int_{\Omega} v u d x-\int_{\Omega} \varphi_{\nu} \phi_{0} v u d x \\
& -\int_{\Omega} \phi_{0} v^{2}\left(\Delta-v^{2}\right)^{-1}\left[\left(\phi_{0}+\varphi_{v}\right) u v\right] d x-\int_{\Omega} f(|x|, v) u d x-\int_{\Omega} g u d x \\
= & \int_{\Omega} \nabla v \nabla u d x+\left(m^{2}-\phi_{0}^{2}\right) \int_{\Omega} v u d x-\int_{\Omega} \varphi_{\nu} \phi_{0} v u d x \\
& -\int_{\Omega}\left(\phi_{0}+\varphi_{v}\right) u v\left(\Delta-v^{2}\right)^{-1}\left[\phi_{0} v^{2}\right] d x-\int_{\Omega} f(|x|, v) u d x-\int_{\Omega} g u d x \\
= & \int_{\Omega} \nabla v \nabla u d x+\int_{\Omega}\left[m^{2}-\left(\phi_{0}+\varphi_{v}\right)^{2}\right] v u d x-\int_{\Omega} f(|x|, v) u d x-\int_{\Omega} g u d x .
\end{aligned}
$$

Hence we get, as an operator in $H^{-1}(\Omega)$,

$$
K^{\prime}(v)=\frac{\partial I}{\partial v}\left(v, \varphi_{v}\right)=-\Delta v+\left[m^{2}-\left(\varphi_{v}+\phi_{0}\right)^{2}\right] v-f(|x|, v)-g .
$$


Lemma 2.2 (Lemma 9 of [11]) For every $u \in H_{0}^{1}(\Omega)$, the function $\varphi_{u}$ satisfies the following inequalities:

$$
-\max \left\{0, \phi_{0}\right\} \leq \varphi_{u} \leq \max \left\{0,-\phi_{0}\right\} \quad \text { a.e. in } \Omega \text {. }
$$

Remark 2.2 In fact, according to (9), we observe that

$$
0 \leq \varphi_{u} \leq-\phi_{0} \quad \text { a.e. in } \Omega
$$

Now we recall Bolle's method for dealing with problems with broken symmetry.

Let $E$ be a Hilbert space equipped with the norm $\|\cdot\|$. Assume that $E=E_{-} \oplus E_{+}$, where $\operatorname{dim}\left(E_{-}\right)<+\infty$, and let $\left(e_{k}\right)_{k \geq 1}$ be an orthonormal base of $E_{+}$. Consider

$$
E_{0}=E_{-}, \quad E_{k+1}=E_{k} \oplus \mathbf{R} e_{k+1}, \quad k \geq 0 .
$$

So $\left\{E_{k}\right\}_{k}$ is an increasing sequence of a finite dimensional subspace of $E$.

Let $I:[0,1] \times E \rightarrow R$ be a $C^{1}$-functional, and set

$$
c_{k}=\inf _{\gamma \in \Gamma} \sup _{v \in E_{k}} I(0, \gamma(v))
$$

with $\Gamma=\{\gamma \in C(E, E): \gamma$ is odd and $\exists R>0$ s.t. $\gamma(v)=v$ for $\|v\| \geq R\}$.

We make the following hypotheses:

$\left(\mathrm{H}_{1}\right)$ I satisfies the following weaker form of the Palais-Smale condition: for every sequence $\left\{\left(\theta_{n}, v_{n}\right)\right\}_{n} \subset[0,1] \times H$ such that

$$
\left\{I\left(\theta_{n}, v_{n}\right)\right\}_{n} \text { are bounded and } \lim _{n \rightarrow+\infty} \frac{\partial I}{\partial \nu}\left(\theta_{n}, v_{n}\right)=0 \text {, }
$$

there is a subsequence converging in $[0,1] \times E$.

$\left(\mathrm{H}_{2}\right)$ For all $b>0$, there exists a constant $C_{b}>0$ such that

$$
|I(\theta, v)|<b \text { implies }\left|\frac{\partial I}{\partial \theta}(\theta, v)\right|<C_{b}\left(\left\|\frac{\partial I}{\partial v}(\theta, v)\right\|+1\right)(\|v\|+1) \text {. }
$$

$\left(\mathrm{H}_{3}\right)$ There exist two continuous maps $\eta_{1}, \eta_{2}:[0,1] \times R \rightarrow R$, which are Lipschitz continuous with respect to the second variable, such that $\eta_{1}(\theta, \cdot) \leq \eta_{2}(\theta, \cdot)$ and such that, for all critical points $v$ of $I(\theta, \cdot)$,

$$
\eta_{1}(\theta, I(\theta, v)) \leq \frac{\partial}{\partial \theta} I(\theta, v) \leq \eta_{2}(\theta, I(\theta, v))
$$

$\left(\mathrm{H}_{4}\right) I(0, v)$ is even and for any finite dimensional space $W$ of $E$, we have

$$
\lim _{\substack{|v| \rightarrow+\infty \\ v \in A}} \sup _{\theta \in[0,1]} I(\theta, v)=-\infty
$$


Let $\chi_{i}:[0,1] \times R \rightarrow R(i=1,2)$ be the flow associated to $\eta_{i}$ that is the solution of problem

$$
\left\{\begin{array}{l}
\frac{\partial}{\partial \theta} \chi_{i}(\theta, s)=\eta_{i}\left(\theta, \chi_{i}(\theta, s)\right) \\
\eta_{i}(0, s)=s
\end{array}\right.
$$

Note that $\chi_{1}$ and $\chi_{2}$ are continuous and that for all $\theta \in[0,1], \chi_{1}(\theta, \cdot)$ and $\chi_{2}(\theta, \cdot)$ they are non-decreasing on $R$. Moreover, since $\eta_{1} \leq \eta_{2}$, we have $\chi_{1} \leq \chi_{2}$. Set

$$
\bar{\eta}_{1}(s)=\sup _{\theta \in[0,1]}\left|\eta_{1}(\theta, s)\right|, \quad \bar{\eta}_{2}(s)=\sup _{\theta \in[0,1]}\left|\eta_{2}(\theta, s)\right| .
$$

In this framework, the following result of Bolle can be proved (for more details and the proof, see [17, Theorem 2.2]).

Theorem 2.1 Assume that $I:[0,1] \times E \rightarrow R$ is $C^{1}$ and satisfies $\left(\mathrm{H}_{1}\right)-\left(\mathrm{H}_{4}\right)$. Then there is $C>0$ such that for every $k$,

(1) either $I(1, x)$ has a critical level $\bar{c}_{k}$ such that $\chi_{2}\left(1, c_{k}\right) \leq \chi_{1}\left(1, c_{k+1}\right) \leq \bar{c}_{k}$,

(2) or $c_{k+1}-c_{k} \leq C\left(\bar{\eta}_{1}\left(c_{k+1}\right)+\bar{\eta}_{2}\left(c_{k}\right)+1\right)$.

Remark 2.3 Note that if $\eta_{2} \leq 0$ in $[0,1] \times R$, then for all $s \in R$, the functional $\chi_{2}(\cdot, s)$ is non-decreasing on $[0,1]$. Hence, in case (1) of the above theorem, we have $c_{k} \leq \bar{c}_{k}$.

\section{Proof of Theorem 1.1}

Now, we consider the family of functionals $J(\theta, u):[0,1] \times H_{0, r}^{1}(\Omega) \rightarrow R$ defined by

$$
J(\theta, u)=J_{0}(u)-\theta \int_{\Omega} g u d x
$$

with

$$
J_{0}(u)=\frac{1}{2} \int_{\Omega}\left(|\nabla u|^{2}+\left[m^{2}-\phi_{0}\left(\varphi_{u}+\phi_{0}\right)\right] u^{2}\right) d x-\int_{\Omega} F(|x|, u) d x .
$$

Clearly, $J$ is a $C^{1}$ functional such that $J(0, u)=J_{0}(u)$ and $J(1, u)=K(u)$. Notice that the functional $J_{0}(u)$ is even by assumption $\left(f_{2}\right)$. According to (14),

$$
\begin{aligned}
\frac{\partial J}{\partial \theta}(\theta, u)= & -\int_{\Omega} g u d x, \\
\frac{\partial J}{\partial u}(\theta, u)[v]= & \int_{\Omega} \nabla v \nabla u d x+\int_{\Omega}\left[m^{2}-\left(\phi_{0}+\varphi_{u}\right)^{2}\right] u v d x \\
& -\int_{\Omega} f(|x|, u) v d x-\theta \int_{\Omega} g v d x
\end{aligned}
$$

for all $\theta \in[0,1]$ and $u, v \in H_{0, r}^{1}(\Omega)$.

The following lemma allows us to prove that functional $J$ verifies the assumption of Bolle's abstract theorem. 
Lemma 3.1 There exist three strictly positive constants $\alpha_{1}, \alpha_{2}$ and $\alpha_{3}$ such that for all $(\theta, u) \in[0,1] \times H_{0, r}^{1}(\Omega)$,

$$
\|\nabla\|_{2}^{2}+\|u\|_{s}^{s} \leq \alpha_{1} J(\theta, u)-\alpha_{2} \frac{\partial J}{\partial u}(\theta, u)[u]+\alpha_{3} .
$$

Proof For any $\delta>0$,

$$
\begin{aligned}
J(\theta, u)-\delta \frac{\partial J}{\partial u}(\theta, u)[u]= & \left(\frac{1}{2}-\delta\right) \int_{\Omega}|\nabla u|^{2} d x+\left(\frac{1}{2}-\delta\right) m^{2} \int_{\Omega} u^{2} d x \\
& +I_{1}+I_{2}-(1-\delta) \theta \int_{\Omega} g u d x
\end{aligned}
$$

with

$$
\begin{aligned}
& I_{1}=-\int_{\Omega}\left[\frac{1}{2} \phi_{0}\left(\varphi_{u}+\phi_{0}\right)-\delta\left(\varphi_{u}+\phi_{0}\right)^{2}\right] u^{2} d x \\
& I_{2}=\int_{\Omega} \delta f(|x|, u) u d x-\int_{\Omega} F(|x|, u) d x
\end{aligned}
$$

By Remark 2.2, we get $\phi_{0} \leq \varphi_{u}+\phi_{0} \leq 0$ and $-\frac{1}{2} \phi_{0} \leq\left(\frac{1}{2}-\delta\right) \phi_{0}-\delta \varphi_{u} \leq\left(\frac{1}{2}-\delta\right) \phi_{0}$. Hence,

$$
\begin{aligned}
I_{1} & =-\int_{\Omega}\left(\varphi_{u}+\phi_{0}\right)\left(\left(\frac{1}{2}-\delta\right) \phi_{0}-\delta \varphi_{u}\right) u^{2} d x \\
& \geq-\frac{1}{2} \int_{\Omega} \phi_{0}^{2} u^{2} d x .
\end{aligned}
$$

By assumption $\left(\mathrm{f}_{3}\right)$, there exist two constants $b_{1}, b_{2}>0$ such that for every $t \in R$,

$$
F(r, u) \geq b_{1}|u|^{s}-b_{2} .
$$

Hence, we get

$$
\begin{aligned}
I_{2} & =\int_{\Omega} \delta f(|x|, u) u d x-\int_{\Omega} F(|x|, u) d x \\
& \geq(s \delta-1) \int_{\Omega} F(|x|, u) d x \\
& \geq(s \delta-1)\left(b_{1}\|u\|_{s}^{s}-m(\Omega) b_{2}\right) .
\end{aligned}
$$

According to Remark 2.1, there exists $\varepsilon_{0}>0$ such that

$$
\left\|\phi_{0}\right\|_{\infty}^{2} \leq\left(\frac{s-2}{s}-\varepsilon_{0}\right) m^{2}
$$

Using the Cauchy-Schwarz inequality, we get

$$
\int_{\Omega} g u d x \leq \frac{\varepsilon_{0} m^{2}}{2}\|u\|_{2}^{2}+\frac{1}{2 \varepsilon_{0} m^{2}}\|g\|_{2}^{2} .
$$


Then equation (23) becomes

$$
\begin{aligned}
& J(\theta, u)-\delta \frac{\partial J}{\partial u}(\theta, u)[u] \\
& \geq\left(\frac{1}{2}-\delta\right) \int_{\Omega}|\nabla u|^{2} d x+\int_{\Omega}\left[\left(\frac{1}{2}-\delta\right) m^{2}-\frac{1}{2} \phi_{0}^{2}-\frac{\varepsilon_{0}}{2} m^{2}\right] u^{2} d x \\
& \quad+(s \delta-1)\left(b_{1}\|u\|_{s}^{s}-m(\Omega) b_{2}\right)-\frac{1}{2 \varepsilon_{0} m^{2}}\|g\|_{2}^{2} .
\end{aligned}
$$

Now, we choose $\delta$ satisfying $\frac{1}{s}<\delta<\frac{1}{s}+\varepsilon_{0}$. Then $s \delta-1>0$ and $\left(\frac{1}{2}-\frac{\varepsilon_{0}}{2}-\delta\right) m^{2}-\frac{1}{2} \phi_{0}^{2}>0$, which implies equation (22).

In the sequel, $C_{i}$ denotes some suitable positive constants. Now, we give the following simple fact.

Lemma 3.2 For any $0 \leq \rho<+\infty$, if the boundary condition $\zeta$ is radial on $\partial \Omega$ in equation (7), then $\phi_{0}$ is also radial in $\Omega$.

Proof (1) Case $\rho=0$, i.e., $\Omega=\Omega_{0}$ is a ball. We denote $h=\zeta(x)$ for $|x|=1$. Then it is obvious that

$$
\phi_{0} \equiv q h-\omega
$$

is the unique solution of the equation

$$
\begin{cases}\Delta \phi_{0}=0 & \text { in } \Omega \\ \phi_{0}=q \zeta-\omega & \text { on } \partial \Omega\end{cases}
$$

(2) Case $\rho>0$, i.e., $\Omega=\Omega_{\rho}$ is an annulus. We denote $h_{1}=\zeta(x)$ for $|x|=\rho$ and $h_{2}=\zeta(x)$ for $|x|=\rho+1$. By calculating using general ordinary differential equation theory and maximal principle, we obtain that the radial function

$$
\phi_{0}(x)=\frac{q\left(h_{1}-h_{2}\right) \rho^{N-2}(\rho+1)^{N-2}}{(\rho+1)^{N-2}-\rho^{N-2}}|x|^{N-2}+\left[q h_{1}-\omega-\frac{q\left(h_{1}-h_{2}\right)(\rho+1)^{N-2}}{(\rho+1)^{N-2}-\rho^{N-2}}\right]
$$

is the unique solution of equation (27).

Proof of Theorem 1.1 We first prove that any critical point $u \in H_{0, r}^{1}(\Omega)$ of $\left.K\right|_{H_{0, r}^{1}(\Omega)}$ is also a critical point of $K$ in $H_{0}^{1}(\Omega)$.

Let $O(N)=\left\{A_{N \times N}\right.$ : orthogonal matrices $\}$. Consider the $O(N)$ group action $T_{g}$ on $L^{2}(\Omega)$ defined by

$$
T_{g} u(x):=u(g x) \quad \text { for any } g \in O(N) \text { and } u \in L^{2}(\Omega) \text {. }
$$

Since for each $u \in H_{0}^{1}(\Omega), \varphi_{u}$ is the unique solution of

$$
\begin{cases}\Delta \varphi_{u}=\left(\varphi_{u}+\phi_{0}\right) u_{0}^{2} & \text { in } \Omega \\ \varphi_{u}=0 & \text { on } \partial \Omega\end{cases}
$$


so, for any $g \in O(N)$, we have $T_{g}\left(\Delta \varphi_{u}\right)=T_{g}\left(\varphi_{u}\right) T_{g}\left(u^{2}\right)+T_{g}\left(\phi_{0}\right) T_{g}\left(u_{0}^{2}\right)$ in $\Omega$. By radial symmetry of $\phi_{0}$ (Lemma 3.2), we get

$$
\begin{cases}\Delta\left(T_{g} \varphi_{u}\right)=T_{g}\left(\varphi_{u}\right)\left(T_{g} u\right)^{2}+\phi_{0} T_{g}\left(u_{0}\right)^{2} & \text { in } \Omega, \\ T_{g} \varphi_{u}=0 & \text { on } \partial \Omega .\end{cases}
$$

Then, by the definition of $\Phi: u \rightarrow \varphi_{u}$, we obtain

$$
T_{g}(\Phi(u))=\Phi\left(T_{g} u\right)
$$

Using (30) and the $T_{g}$ invariance of the norm in $H_{r}^{1}(\Omega), L^{p}(\Omega)$, we deduce that $K$ is $O(N)$ invariant, i.e.,

$$
K\left(T_{g} u\right)=K(u), \quad \forall u \in H_{0}^{1}(\Omega), g \in O(N) .
$$

Then, according to the principle of symmetric criticality [18], any critical point $u \in$ $H_{0, r}^{1}(\Omega)$ of $\left.K\right|_{H_{0, r}^{1}(\Omega)}$ is also a critical point of $K$ in $H_{0}^{1}(\Omega)$. Then we want to apply Bolle's method to $K$ restricted to $H_{0, r}^{1}(\Omega)$.

Firstly, we prove that $J$ satisfies assumption $\left(\mathrm{H}_{1}\right)$ of Theorem 2.1. So let us consider a sequence $\left\{\left(\theta_{n}, u_{n}\right)\right\} \subset[0,1] \times H_{0, r}^{1}(\Omega)$ such that

$$
\left\{J\left(\theta_{n}, u_{n}\right)\right\}_{n} \text { is bounded and } \lim _{n \rightarrow \infty} \frac{\partial J}{\partial u}\left(\theta_{n}, u_{n}\right)=0
$$

Let $\varphi_{n}=\Phi\left[u_{n}\right]$. Then the expression of $\frac{\partial J}{\partial u}$ and (15) implies

$$
\varepsilon_{n}=\frac{\partial J}{\partial u}\left(\theta_{n}, u_{n}\right)=-\Delta u_{n}+\left[m^{2}-\left(\varphi_{n}+\phi_{0}\right)^{2}\right] u_{n}-f\left(|x|, u_{n}\right)-\theta g
$$

with $\varepsilon \rightarrow 0$. Clearly, Lemma 3.1 implies $\left\{\left\|\nabla u_{n}\right\|\right\}_{n}$ is bounded. By assumption $\left(\mathrm{f}_{1}\right)$ and $L^{p}(\Omega) \hookrightarrow H_{0, r}^{1}(\Omega)$, we get

$$
\int_{\Omega}\left|f\left(r, u_{n}\right)\right|^{\frac{p}{p-1}} d x \leq C_{1}\left(\left\|u_{n}\right\|_{p}^{p}+1\right) \leq C_{2}\left(\|\nabla u\|_{2}^{p}+1\right) .
$$

That is, $\left\{f\left(r, u_{n}\right)\right\}_{n}$ are bounded in $L^{\frac{p}{p-1}}(\Omega)$. Moreover, by Remark 2.2, $\left\{\left[m^{2}-\left(\varphi_{n}+\phi_{0}\right)^{2}\right] u_{n}\right\}_{n}$ are bounded in $L^{2}(\Omega)$. Hence these two sequences $\left\{f\left(r, u_{n}\right)\right\}_{n}$ and $\left\{\left[m^{2}-\left(\varphi_{n}+\phi_{0}\right)^{2}\right] u_{n}\right\}_{n}$, up to subsequences, converge in $H_{r}^{-1}(\Omega)$ (the dual space of $H_{0, r}^{1}(\Omega)$ ). Thus, as $-\Delta$ is an isomorphism from $H_{0, r}^{1}(\Omega)$ to its dual space $H_{r}^{-1}(\Omega)$, there exists a subsequence of $\left\{u_{n}\right\}$ converging strongly in $H_{0, r}^{1}(\Omega)$ by the standard argument.

By equation (20), for all $(\theta, u) \in[0,1] \times H_{0, r}^{1}(\Omega)$,

$$
\left|\frac{\partial J}{\partial \theta}(\theta, u)\right| \leq\|g\|_{2}\|u\|_{2} \leq\|g\|_{2}\|\nabla u\|_{2} .
$$

Hence Lemma 3.1 implies that $J$ satisfies assumption $\left(\mathrm{H}_{2}\right)$ of Theorem 2.1.

$$
\text { If }(\theta, u) \in[0,1] \times H_{0, r}^{1}(\Omega) \text { satisfies } \frac{\partial J}{\partial u}(\theta, u)=0 \text {, then }
$$

$$
\|u\|_{s}^{s} \leq \alpha_{1}|J(\theta, u)|+\alpha_{3} \leq \alpha_{4}\left(1+J^{2}(\theta, u)\right)^{\frac{1}{2}},
$$


with $\alpha_{4}=\sqrt{\left(\alpha_{1}+\alpha_{3}\right) \max \left\{\alpha_{1}, \alpha_{3}\right\}}$. So, there exists a positive constant $C_{3}$ such that

$$
\left|\frac{\partial J}{\partial \theta}(\theta, u)\right| \leq C_{3}\left(1+J^{2}(\theta, u)\right)^{\frac{1}{2 s}}
$$

by (33). Hence, $J$ verifies assumption $\left(\mathrm{H}_{3}\right)$ of Theorem 2.1 with $\eta_{2}(\theta, t)=-\eta_{1}(\theta, t)=C_{3}(1+$ $\left.t^{2}\right)^{\frac{1}{2 s}}$. Notice that $\bar{\eta}_{1}(t)=\bar{\eta}_{2}(t)=C_{3}\left(1+t^{2}\right)^{\frac{1}{2 s}}$. So, by the definition of $J$,

$$
\begin{aligned}
J(\theta, u) & \leq \frac{1}{2}\|\nabla u\|_{2}^{2}+C_{4}\|u\|_{2}^{2}-\int_{\Omega} F(|x|, u) d x-\theta \int_{\Omega} g u d x \\
& \leq \frac{1}{2}\|\nabla u\|_{2}^{2}+C_{5}\|u\|_{2}^{2}-b_{1}\|u\|_{s}^{s}-b_{2} m(\Omega)-\|g\|_{2}^{2},
\end{aligned}
$$

using inequalities (25) and (33). Then, as in a finite dimensional space all the norms are equivalent and $s>2$, we get that $J$ satisfies assumption $\left(\mathrm{H}_{4}\right)$ of Theorem 2.1.

Now we apply Theorem 2.1 to $J$ and assume that case (1) in the results of Theorem 2.1 occurs for all $k$ large enough. Then, according to the definition of $\bar{\eta}_{1}$ and $\bar{\eta}_{2}$, we obtain

$$
c_{k+1}-c_{k} \leq C_{6}\left(c_{k+1}^{\frac{1}{s}}+c_{k}^{\frac{1}{s}}+1\right),
$$

where $c_{k}$ is the critical level of $J$ defined by (17). Hence, using a similar argument as in Lemma 5.3 of [19], there exists an integer $k_{0} \in N$ such that

$$
c_{k} \leq C_{7} k^{\frac{s}{s-1}} \quad \text { for all } k \geq k_{0} .
$$

On the other hand, assumption $\left(f_{1}\right)$ implies

$$
J(0, u) \geq \frac{1}{2}\|\nabla u\|_{2}^{2}-C_{8}\|u\|_{p}^{p}-C_{9}
$$

So, by applying the arguments developed in [20], the radial symmetry of the problem implies

$$
c_{k} \geq C_{10} k^{\frac{p}{p-2}}, \quad \text { if } k \text { is large enough, }
$$

which contradicts with (36).

That means case (1) in the result of Theorem 2.1 occurs for infinitely many indexes, and a sequence $\left\{\bar{c}_{n}\right\}$ of the critical level of $J(1, \cdot)=K(\cdot)$ exists such that $\bar{c}_{k} \rightarrow+\infty$ by Remark 2.3 and (38). Thus, if $\left\{u_{n}\right\}_{n}$ is the corresponding sequence of critical points, by (34) for $\theta=1$, it follows that $\left\|\nabla u_{n}\right\|_{2} \rightarrow+\infty$. We complete the proof of Theorem 1.1. 


\section{Acknowledgements}

This work is supported by the National Natural Science Foundation of China (Grant 11226128).

Received: 17 May 2013 Accepted: 11 November 2013 Published: 13 Dec 2013

\section{References}

1. D'Aprile, T, Mugnai, D: Solitary waves for nonlinear Klein-Gordon-maxwell and Schrodinger-maxwell equations. Proc. R. Soc. Edinb., Sect. A 134, 893-906 (2004)

2. Benci, V, Fortunato, D: The nonlinear Klein-Gordon equation coupled with the Maxwell equations. Nonlinear Anal. 47, 6065-6072 (2001)

3. Benci, V, Fortunato, D: Solitary waves of the nonlinear Klein-Gordon equation coupled with the Maxwell equations. Rev. Math. Phys. 14, 409-420 (2002)

4. D’Aprile, T, Mugnai, D: Non-existence results for the coupled Klein-Gordon-Maxwell equations. Adv. Nonlinear Stud. 4 307-322 (2004)

5. Cassani, D: Existence and non-existence of solitary waves for the critical Klein-Gordon equation coupled with Maxwell's equations. Nonlinear Anal. 58, 733-747 (2004)

6. Carrião, P, Cunha, P, Miyagaki, O: Positive and ground state solutions for the critical Klein-Gordon-Maxwell system with potentials. Nonlinear Anal. TMA 75, 4068-4078 (2012)

7. Azzollini, A, Pomponio, A: Ground state solutions for the nonlinear Klein-Gordon-Maxwell equations. Topol. Methods Nonlinear Anal. 35, 33-42 (2010)

8. Carrião, P, Cunha, P, Miyagaki, O: Existence results for the Klein-Gordon-Maxwell equations in higher dimensions with critical exponents. Commun. Pure Appl. Anal. 10, 709-718 (2011)

9. Georgiev, V, Visciglia, N: Solitary waves for Klein-Gordon-Maxwell system with external Coulomb potential. J. Math. Pures Appl. 84, 957-983 (2005)

10. Mugnai, D: Coupled Klein-Gordon and Born-Infeld-type equations: looking for solitary waves. Proc. R. Soc. Lond. Ser. A 460, 1519-1527 (2004)

11. D'Avenia, P, Pisani, L, Siciliano, G: Dirichlet and Neumann problems for Klein-Gordon-Maxwell systems. Nonlinear Anal. 71, 1985-1995 (2009)

12. D'Avenia, P, Pisani, L, Siciliano, G: Klein-Gordon-Maxwell systems in a bounded domain. Discrete Contin. Dyn. Syst. 26, 135-149 (2010)

13. Chen, SJ, Tang, CL: Multiple solutions for nonhomogeneous Schrödinger-Maxwell and Klein-Gordon-Maxwell equations on $R^{3}$. NoDEA Nonlinear Differ. Equ. Appl. 17, 559-574 (2010)

14. Candela, AM, Salvatore, A: Multiple solitary waves for non-homogeneous Schrödinger-Maxwell equations. Mediterr. J. Math. 3(3-4), 483-493 (2006)

15. Candela, AM, Salvatore, A: Multiple solitary waves for non-homogeneous Klein-Gordon-Maxwell equations. In: More Progresses in Analysis, pp. 753-762 (2009)

16. Bolle, P: On the Bolza problem. J. Differ. Equ. 152, 274-288 (1999)

17. Bolle, $\mathrm{P}, \mathrm{Ghoussoub}, \mathrm{N}$, Tehrani, $\mathrm{H}$ : The multiplicity of solutions in nonhomogeneous boundary value problems. Manuscr. Math. 101, 325-350 (2000)

18. Palais, RS: The principle of symmetric criticality. Commun. Math. Phys. 69, 19-30 (1979)

19. Bahri, A, Berestycki, H: A perturbation method in critical point theory and applications. Trans. Am. Math. Soc. 267, 1-32 (1981)

20. Candela, AM, Palmieri, G, Salvatore, A: Radial solutions of semilinear elliptic equations with broken symmetry. Topol. Methods Nonlinear Anal. 27, 117-132 (2006)

10.1186/1029-242X-2013-583

Cite this article as: Wu and Ge: A multiplicity result for the non-homogeneous Klein-Gordon-Maxwell system in rotationally symmetric bounded domains. Journal of Inequalities and Applications 2013, 2013:583

\section{Submit your manuscript to a SpringerOpen ${ }^{\ominus}$ journal and benefit from:}

- Convenient online submission

Rigorous peer review

- Immediate publication on acceptance

- Open access: articles freely available online

- High visibility within the field

- Retaining the copyright to your article 